Document downloaded from:

http://hdl.handle.net/10251/80624

This paper must be cited as:

Pedroche Sánchez, F.; Romance, M.; Criado Herrero, R. (2016). A biplex approach to PageRank centrality: from classic to multiplex networks. Chaos. 26(6):065301-1-065301-9. doi:10.1063/1.4952955.

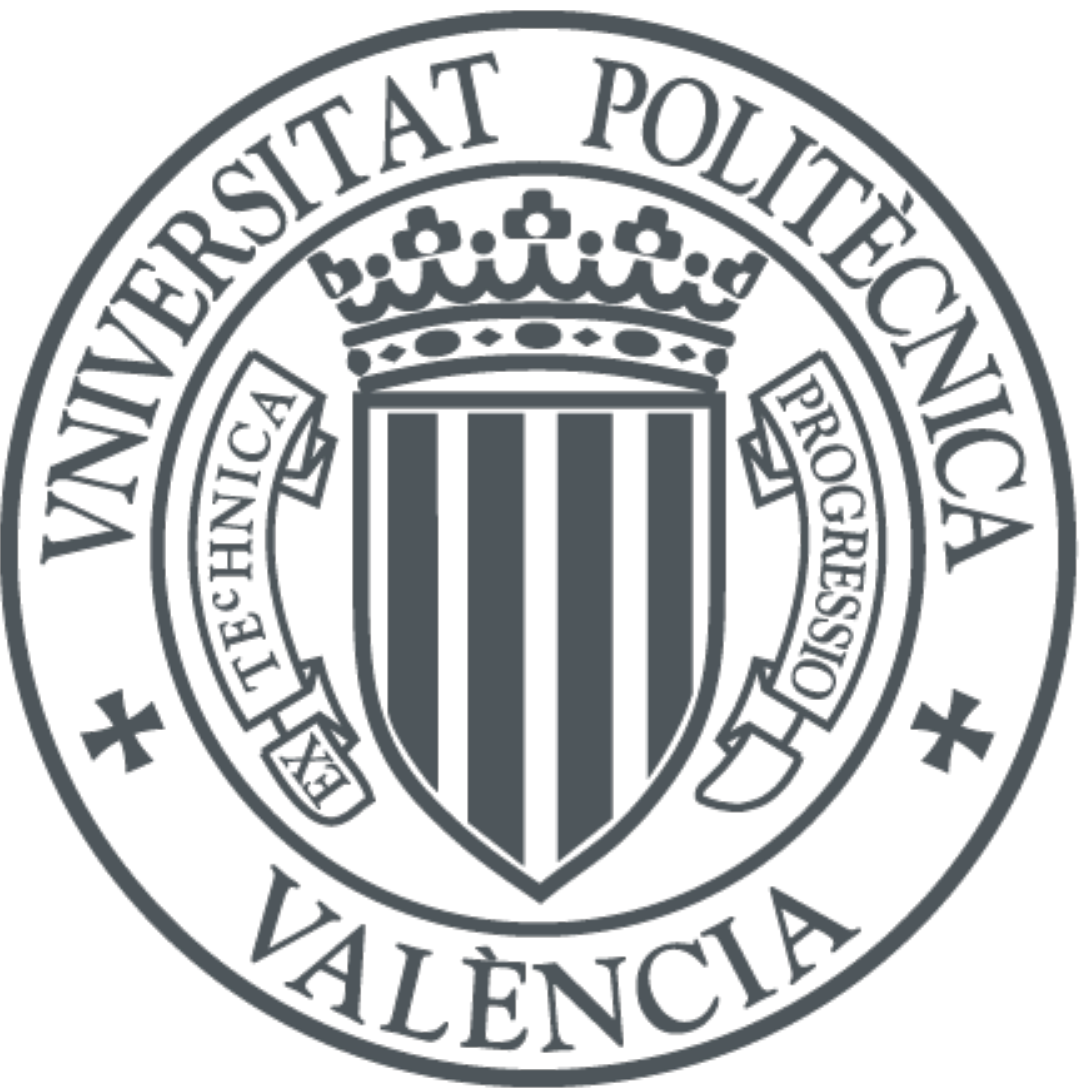

The final publication is available at

http://dx.doi.org/10.1063/1.4952955

Copyright American Institute of Physics

Additional Information 


\title{
A biplex approach to PageRank centrality: From classic to multiplex networks
}

\author{
Francisco Pedroche, ${ }^{1}$ Miguel Romance, ${ }^{2,3}$ and Regino Criado ${ }^{2,3}$ \\ ${ }^{1}$ Institut de Matemàtica Multidisciplinària, Universitat Politècnica de València, Valencia (Spain) \\ ${ }^{2}$ Department of Applied Mathematics, Rey Juan Carlos University, Madrid (Spain) \\ ${ }^{3}$ Center for Biomedical Technology (CTB), Technical University of Madrid (Spain)
}

(Dated: February 29, 2016)

\begin{abstract}
In this paper we present a new view of the PageRank algorithm inspired by multiplex networks. This new approach allows to introduce a new centrality measure for classic complex networks and a new proposal to extend the usual PageRank algorithm to multiplex networks. We give some analytical relations between these new approaches and the classic PageRank centrality measure and we illustrate the new parameters presented by computing them on real underground networks.
\end{abstract}

One major issue in science, from biological systems to information networks and many other fields, is determining the most relevant elements in a given complex system. Centrality measures help spotting the most important nodes of a complex networks by giving a value to each vertex in the system. There are many different centrality measures in Networks Science, including local parameter (such as the in-degree), metric parameters (such as the betweenness centrality) and spectral centralities (such as the eigenvector centrality), but the PageRank centrality plays a relevant role, since it has many relevant applications. This measure is the basic ingredient of the (probably) most famous web searcher (Google), but it also has many applications to different reallife problems, ranging from biological systems to cibersecurity (hacking detection). In this paper we discuss about a biplex approach to the classic PageRank algorithm and how this new insight allows to introduce new centrality measures on complex and multiplex networks that can be useful for ranking the elements of a complex system according to their relevance.

\section{INTRODUCTION AND NOTATION}

Understanding the specific role of each element in a complex system and its relevance in the whole structure is the first step to understand the behaviour of the system. There are many functions in the Network's Science literature that try to give a precise and quantitative measure of the relevance of the nodes in a network [2]. Social Network Analysis has a long tradition in measuring the importance of each actor in a social system by means of the so called centrality measures [26], but the range and applications of these measures go beyond Social Networks and it can be successfully applied to any complex network. Centrality measures are designed to rank nodes based on their structural position inside the network, but there is no big consensus about the best centrality measure, since different measures must be considered in order to analyse different contexts [2]. From degree based to spectral measures going through metric-based measures and others, there are plenty of different approaches to the analysis of relevance in a network, but the PageRank centrality [19] is a highlight since it is the basic ingredient in web information in general and in Google's web searcher in particular.

The main goal of this paper is introducing a new approach to the PageRank analysis that can be used to define new centrality measures on complex networks and multiplex networks. During the last years the Network's Science scientific community has realized that many reallife systems must be modelled by taking into account the fact that the interrelations between nodes are heterogeneous [1, 15]. This heterogeneity makes that some structural and dynamical properties emerge from the distinction between different kinds of links and the new models of multilayer and multiplex networks have been introduced [1, 6, 15, 23]. The introduction of this new multilayer paradigm requires a revision of all the structural techniques and tools previously developed for (classic) complex network (also called monoplex network in this new language), and therefore the centrality measures must be revisited from this new point of view [9, 14, 22].

This paper presents a new proposal that starts by defining an approximation of usual PageRank in graphs considered as a two-layer approach. It is shown that this approximation, that we call $\hat{\pi}_{A}$, is exact in some instances and is a very good approximation in same test examples. Furthermore, this new approach can be used for introduting a new model for PageRank of a multiplex network that uses the underlying ideas in $\hat{\pi}_{A}$. There are some different extensions of the PageRank for multiplex networks [1, 9, 14], but the key point of the new approach presented is that we can associate two layers to each real layer of the multiplex and by using this approach we are capable of defining a PageRank-like model to the whole multiplex. It is shown that some different approaches can be defined, and a simplified mathematical formalism is included that allows extending the model for multiplex networks with any number of layers.

Let us introduce the basic notation used. Along the paper we will recall some notation from [13]. Let $\mathcal{G}=$ $(\mathcal{N}, \mathcal{E})$ be a directed graph where $\mathcal{N}=\{1,2, \ldots, n\}$ and $n \in \mathbb{N}$. The link $(i, j)$ belongs to the set $\mathcal{E}$ if and only 
if there exists a link connecting node $i$ to node $j$. The adjacency matrix of $\mathcal{G}$ is an $n \times n$-matrix

$$
A=\left(a_{i j}\right) \text { where } a_{i j}= \begin{cases}1, & \text { if }(i, j) \text { is a link of } \mathcal{G} \\ 0, & \text { otherwise }\end{cases}
$$

A link $(i, j)$ is said to be an outlink for node $i$ and an $i n$ link for node $j$. We denote $k_{\text {out }}(i)$ the outdegree of node $i$, i.e., the number of outlinks of a node $i$. Notice that $k_{\text {out }}(i)=\sum_{k} a_{i k}$. The graph $\mathcal{G}=(\mathcal{N}, \mathcal{E})$ may have dangling nodes, which are nodes $i \in \mathcal{N}$ with zero outdegree.

Let $P_{A}=\left(p_{i j}\right) \in \mathbb{R}^{n \times n}$ be the row stochastic matrix associated to $\mathcal{G}$ defined in the following way:

- if $i$ is a dangling node, $p_{i j}=0$ for all $j=1, \ldots, n$,

- otherwise, $p_{i j}=\frac{a_{i j}}{k_{o u t}(i)}=\frac{a_{i j}}{\sum_{k} a_{i k}}$.

Vectors of $\mathbb{R}^{n}$ will be denoted by column matrices. In particular, $\mathbf{e}=(1, \cdots, 1)^{T}$.

The Google matrix $G=G(\alpha, \mathbf{u}, \mathbf{v}), \alpha \in(0,1)$ (called damping factor), with dangling nodes and personalized vector $\mathbf{v}$ is defined as

$$
G=\alpha\left(P_{A}+\mathbf{d u}^{T}\right)+(1-\alpha) \mathbf{e v}^{T} \in \mathbb{R}^{n \times n}
$$

(this matrix $G$ is row-stochastic, i.e., $G \mathbf{e}=\mathbf{e}$ ). We recall that the distribution of dangling nodes is given by $\mathbf{u} \in \mathbb{R}^{n}$ such that $\mathbf{u}>0$ and $\mathbf{u}^{T} \mathbf{e}=1$. The dangling nodes will be characterized by a vector $\mathbf{d} \in \mathbb{R}^{n}$ defined as $\mathbf{d}=$ $\left(d_{1}, \ldots, d_{n}\right)^{T}$, where

$$
d_{i}= \begin{cases}1, & \text { if } i \text { is a dangling node of } \mathcal{G} \\ 0, & \text { otherwise. }\end{cases}
$$

The PageRank vector $\pi=\pi(\alpha, \mathbf{u}, \mathbf{v})$ is the unique eigenvector of $G^{T}$ associated to eigenvalue 1 such that $\pi^{T} \mathbf{e}=$ 1, i.e., $\pi>0, \pi^{T} \mathbf{e}=1$ and $\pi^{T} G=\pi^{T}$ (see [19]). Recall also that

$$
\pi^{T}=\alpha \pi^{T}\left(P_{A}+\mathbf{d} \mathbf{u}^{T}\right)+(1-\alpha) \mathbf{v}^{T}
$$

We will write $\pi_{A}^{T}$ when needed. We shall assume that there are no dangling nodes in the rest of the paper, that is $\mathbf{d}=\mathbf{0}$, and therefore $P_{A}$ is row stochastic, but if there were dangling nodes we would work with $P_{A}+\mathbf{d} \mathbf{u}^{T}$ and it is easy to check that the results throughout the paper also will hold with some straightforward modifications.

The structure of the paper is the following: After this introduction, Section $\amalg$ is devoted to present the Biplex approach for the usual PageRank of a complex (monoplex) network. By using this approach, a new centrality measure is given for complex (monoplex) networks and the relationship between this new measure and the usual PageRank is analysed theoretically (with some analytical results) and through some real examples (by computing some linear correlations). Section III includes the use of the Biplex approach for giving a PageRank centrality for multiplex networks. For the sake of clarity, the case of multiplex networks with two layers (biplex networks) is developed, giving later the model for general multiplex network. Some analytical results are included that relate this new centrality measures with the usual PageRank. In order to illustrate the use, similarities and differences of this new multiplex PageRank with the usual one, Section $[\mathrm{IV}$ considers the Madrid underground transportation system (Metro of Madrid) as a multiplex network with as many layers as lines this underground system has (12). In this Section several numerical computations are presented in order to highlight the fact that the new multiplex PageRank is a new centrality measure for multiplex networks.

\section{BIPLEX APPROACHES FOR CLASSIC PAGERANK}

Roughly speaking, the (classic) PageRank of a complex network $\mathcal{G}$ is the frequency vector of a random walker that can jump between nodes following to basic principles: on the one hand, the walker can use the local links given by the network $\mathcal{G}$ (that we call a physical walk) and, on the other hand, the walker can jump to any other node in the network according to the personalized vector $\mathbf{v}$ (called a teleportation). Hence, the PageRank of $\mathcal{G}$ with personalized vector $\mathbf{v}$ and damping factor $\alpha \in(0,1)$ can be understood as the stationary distribution of a Markov chain that occurs in a (weighted) multilayer network $B(\mathcal{G})$ with the same set of nodes of $\mathcal{G}$ and two layers $\ell_{1}$ and $\ell_{2}$ (biplex network) such that:

- $\ell_{1}$ (that we call the physical layer) is exactly the network $\mathcal{G}$,

- $\ell_{2}$ (that we call the teleportation layer) is a all-toall network, with weights given by the personalized vector $\mathbf{v}=\left(v_{1}, \cdots, v_{n}\right)^{T}$, that is the adjacency matrix of $\ell_{2}$ is $\mathbf{e v}^{T}$.

Once we have defined $B(\mathcal{G})$, the (classic) PageRank of $\mathcal{G}$ is the stationary distribution of a Markov chain on $B(\mathcal{G})$ modeled as follows:

1. We choose a node $x_{0}$ of $\ell_{1}$ and the walker starts the stochastic process at this node.

2. At the beginning of each round $t$, with probability $\alpha$ the walker will move on layer $\ell_{1}$ and with probability $1-\alpha$ the walker will move on layer $\ell_{2}$ according to the following rules:

- If the walker moves on the physical layer $\ell_{1}$, it follows the usual rules for a (uniform) random walker on $\ell_{1}$.

- If the walker moves on the teleportation layer $\ell_{2}$, it follows the rules for a biased random walker on a complete graph with transition matrix $\mathbf{e v}^{T}$. 


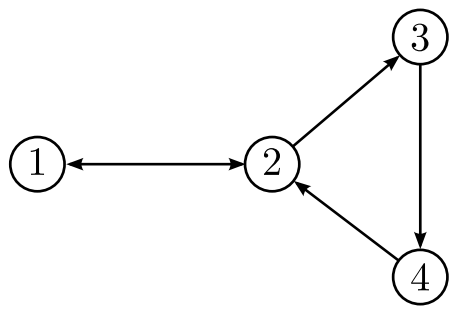

(a)

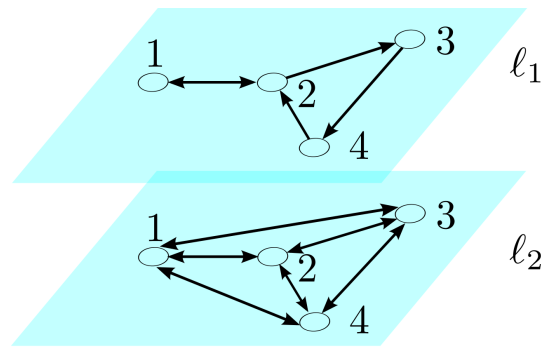

(b)

FIG. 1. The (classic) PageRank of a network as a random walker on a biplex multilayer network.

The mathematical model for this multiplex stochastic process can be written in terms of (block) supraadjacency matrices (see, for example, the supplementary material in 7] or 21]) such that the (classic) PageRank of $\mathcal{G}$ is obtained from the unique, positive and normalized eigenvector of the block matrix

$$
M_{P R}=\left(\begin{array}{cc}
\alpha P_{A} & (1-\alpha) \mathbf{e v}^{T} \\
\alpha P_{A} & (1-\alpha) \mathbf{e v}^{T}
\end{array}\right) \in \mathbb{R}^{2 n \times 2 n} .
$$

As we stated in the Introduction, we shall assume that there are no dangling nodes, that is $\mathbf{d}=\mathbf{0}$, and therefore $P_{A}$ is row stochastic, but if there were dangling nodes we would work with $P_{A}+\mathbf{d} \mathbf{u}^{T}$ and the results throughout the paper also will hold. It is straightforward to check that there is a unique normalized and positive eigenvector $\tilde{\pi}^{T}=\left[\begin{array}{ll}\pi_{1}^{T} & \pi_{2}^{T}\end{array}\right] \in \mathbb{R}^{2 n}$ of $M_{P R}$. Furthermore, it is easy to show that the (classic) PageRank $\pi$ of $\mathcal{G}$ can be written as $\pi=\pi_{1}+\pi_{2}$

By using this multilayer approach of the PageRank, matrix $M_{P R}$ corresponds to the supra-adjacency matrix of a multilayer network with two layer that have crosslayer connections 1]. Figure 1 presents the multilayer network associated to a four-noded complex network. The main advantage of this model is the fact that the (classic) PageRank of a complex network is obtained as the stationary distribution of a random walker in a multilayer network with two layers (one corresponding to the physical walker and other to the teleportation walker, following the two basic principles of the PageRank), but this approach has two main drawbacks: on the one hand, $M_{P R}$ corresponds to the supra-adjacency matrix of a multilayer network with non-trivial cross-layer connections, which makes that it is not a multiplex network if $P_{A} \neq I \neq \mathbf{e v}^{T}$. On the other hand, since the nondiagonal blocks are different in general, the transitions between the two layers are non-symmetric.

In order to avoid these drawbacks, if we take the block matrix

$$
M_{A}=\left(\begin{array}{cc}
\alpha P_{A} & (1-\alpha) I \\
\alpha I & (1-\alpha) \mathbf{e v}^{T}
\end{array}\right) \in \mathbb{R}^{2 n \times 2 n}
$$

with $I$ the identity matrix of size $n$, then $M_{A}$ defines a Markov chain in a multiplex network with two layers and therefore there always exists a unique vector $\hat{\pi}_{M} \in \mathbb{R}^{2 n}$ such that:

(i) $\hat{\pi}_{M}^{T}=\hat{\pi}_{M}^{T} M_{A}$ with $\hat{\pi}_{M}^{T} \mathbf{e}=1$.

(ii) If $\hat{\pi}_{M}^{T}=\left[\begin{array}{ll}\pi_{u}^{T} & \pi_{d}^{T}\end{array}\right] \in \mathbb{R}^{2 n}$ with $\pi_{u}, \pi_{d} \in \mathbb{R}^{n}$, then $\pi_{u}^{T} \mathbf{e}=\alpha$ and $\pi_{d}^{T} \mathbf{e}=1-\alpha$.

It is important to remark that either $M_{P R}$ and $M_{A}$ correspond to the supra-adjacency matrices of two multilayer networks with the same two layers, but they differ in the cross-connections between the layers. In fact, while the multilayer network associated to $M_{A}$ is a multiplex network, this is not the case of the multilayer network associated to $M_{P R}$.

Note that $(i)$ is consequence of the fact that $M_{A}$ is irreducible since $\mathbf{e v}^{T}$ is a positive matrix and $M_{A}$ is also primitive since $M_{A}$ has some non-zero diagonal elements (by using [25]). Hence, by using Perron-Frobenius theorem, $\hat{\pi}_{M}^{T}$ is the only eigenvector associated to the unit eigenvalue, which is the spectral radius, and there is no other eigenvalue with absolute value 1 .

Since $\hat{\pi}_{M}^{T}$ is unique we get that $\pi_{u}^{T}$ and $\pi_{d}^{T}$ are unique vectors and therefore if we want to show (ii), we only have to prove that $\pi_{u}^{T} \mathbf{e}=\alpha$. Note that

$$
\begin{aligned}
& \hat{\pi}_{M}^{T}=\left[\begin{array}{ll}
\pi_{u}^{T} & \pi_{d}^{T}
\end{array}\right]=\left[\begin{array}{ll}
\pi_{u}^{T} & \pi_{d}^{T}
\end{array}\right]\left(\begin{array}{cc}
\alpha P_{A} & (1-\alpha) I \\
\alpha I & (1-\alpha) \mathbf{e v}^{T}
\end{array}\right) \\
& =\left[\pi_{u}^{T} \alpha P_{A}+\pi_{d}^{T} \alpha \mid(1-\alpha) \pi_{u}^{T}+(1-\alpha) \pi_{d}^{T} \mathbf{e v}^{T}\right],
\end{aligned}
$$

that is

$$
\left.\begin{array}{l}
\pi_{u}^{T}=\pi_{u}^{T} \alpha P_{A}+\pi_{d}^{T} \alpha \\
\pi_{d}^{T}=(1-\alpha) \pi_{u}^{T}+(1-\alpha) \pi_{d}^{T} \mathbf{e v}^{T}
\end{array}\right\} .
$$

Now, if we assume that $\pi_{u}^{T} \mathbf{e}=\mu$, then $\pi_{d}^{T} \mathbf{e}=1-\mu$ and hence

$$
\left.\begin{array}{l}
\pi_{u}^{T}=\pi_{u}^{T} \alpha P_{A}+\pi_{d}^{T} \alpha \\
\pi_{d}^{T}=(1-\alpha) \pi_{u}^{T}+(1-\alpha)(1-\mu) \mathbf{v}^{T}
\end{array}\right\}
$$

Finally, by multiplying the first equation of this system by e we get that

$$
\pi_{u}^{T} \mathbf{e}=\pi_{u}^{T} \alpha P_{A} \mathbf{e}+\pi_{d}^{T} \mathbf{e} \alpha,
$$

so $\mu=\alpha \mu+(1-\mu) \alpha$ and we conclude that $\mu=\alpha$. 
Properties (i) and (ii) stated before suggest considering a new PageRank-like centrality of a complex network by using this new biplex approach as the following definition states:

Definition 2.1 Given a complex network $\mathcal{G}$ with $n \in \mathbb{N}$ nodes and whose adjacency matrix is $A$, the two-layer approach PageRank of $A$ is the vector

$$
\hat{\pi}_{A}=\pi_{u}+\pi_{d} \in \mathbb{R}^{n},
$$

where $\left[\begin{array}{ll}\pi_{u}^{T} & \pi_{d}^{T}\end{array}\right]^{T} \in \mathbb{R}^{2 n}$ is the unique normalized and positive eigenvector of matrix $M_{A}$ given in (4).

The existence and uniqueness of this new centrality measure is always guaranteed by Perron-Frobenius' theorem (as we pointed out before), but it is natural to ask if it is correlated with the classic PageRank, since both measures comes from two similar block matrices. The following results states that if we consider undirected regular graphs, both measures coincide.

Theorem 2.2 Let $\mathcal{G}=(\mathcal{N}, \mathcal{E})$ be an undirected network with $n \in \mathbb{N}$ nodes. if we take $\mathbf{d}=\mathbf{0}, \mathbf{v}=\mathbf{e} / n \in \mathbb{R}^{n}$ and $k_{\text {out }}(i)=k_{\text {out }}(j)$ for all $i, j \in \mathcal{N}$ (i.e. $\mathcal{G}$ is regular), then $\hat{\pi}_{A}=\pi_{A}$.

Proof:

Let us begin by showing that $\pi_{A}=\mathbf{e} / n$. Note first that since $k_{\text {out }}(i)=k_{\text {out }}(j), \forall i, j \in \mathcal{N}$ and $A=A^{T}$, we have that $P_{A}$ is symmetric and therefore column stochastic. By plugging $\pi_{A}=\mathbf{e} / n, \mathbf{v}=\mathbf{e} / n$ and $\mathbf{d}=\mathbf{0}$, into equation (2), we get that

$$
\frac{1}{n} \mathbf{e}^{T}=\frac{\alpha}{n} \mathbf{e}^{T} P_{A}+\frac{1-\alpha}{n} \mathbf{e}^{T},
$$

which holds since $P_{A}$ is column stochastic, that makes that $\mathbf{e}^{T} P_{A}=\mathbf{e}^{T}$. Finally, in order to show that $\hat{\pi}_{A}=$ $\mathbf{e} / n$, if we take $\pi_{u}=\alpha \mathbf{e} / n$ and $\pi_{d}=(1-\alpha) \mathbf{e} / n$, then by using the same argument as above it is easy to see that equation (5) holds and therefore $\hat{\pi}_{A}=\pi_{u}+\pi_{d}=\mathbf{e} / n$.

Note that the last theorem covers a large quantity of situations, including the extreme case of complete graphs. Furthermore, taking into account that for large networks a good correlation usually exists between indegree and PageRank (see [12]) one expects the twolayer approach PageRank to be a good approximation to PageRank in most instances. In order to numerically confirm this idea, Table I shows some linear regression results for the two-layer approach PageRank versus the usual PageRank in some real networks. In particular the network of Facebook's users from the California Institute of Technology [24] (denoted as FB), the giant component of the network of users of the Pretty-Good-Privacy algorithm for secure information interchange [3] (denoted as PGP) and the Stanford's network [4, 16] (denoted as Stanford) has been considered. The numerical correlation between $\hat{\pi}_{A}$ and $\pi_{A}$ for those real networks presented in Table@supports that these measures are strongly correlated but different in general for a non-regular complex network.

\begin{tabular}{|l|r|l|}
\hline Network & $n$ & $r^{2}$ \\
\hline FB & 769 & 0.99 \\
\hline PGP & 10680 & 0.92 \\
\hline Stanford & 50000 & 0.93 \\
\hline
\end{tabular}

TABLE I. Linear correlation coefficient $r^{2}$ for $\hat{\pi}_{A}$ versus $\pi_{A}$ in some numerical tests for complex networks with $n$ nodes.

\section{MULTILAYER APPROACH FOR PAGERANK OF MULTIPLEX NETWORKS}

The biplex approaches for complex networks presented in the previous section allow also to define new centrality measures on multiplex network. There are several alternative definitions of the PageRank centrality for multiplex networks [14, 23] and other spectral-based and random-walkers based measures [9, 21, 22] but the biplex approach can be extended for multiplex network in order to give a new point of view of these measures.

Given a multiplex network $\mathcal{M}=(\mathcal{N}, \mathcal{E}, \mathcal{S})$, with layers $\mathcal{S}=\left\{\ell_{1}, \cdots, \ell_{k}\right\}$, a new multiplex PageRank centrality can be defined by using the following basic idea: associating to each layer $\ell_{i}$ a two-layer random walker with one the physical layer (with topology given by $\ell_{i}$ ) and a teleportation layer $\mathbf{e v}_{i}^{T}$. In addition to this, transition between teleportation layers of different real layers and between physical layers must be allowed. For the sake of clarity, we will start by defining this measure for a multiplex network $\mathcal{M}$ with two layers.

If we take a multiplex network $\mathcal{M}=(\mathcal{N}, \mathcal{E}, \mathcal{S})$, with layers $\mathcal{S}=\left\{\ell_{1}, \ell_{2}\right\}$ whose adjacency matrices are $A_{1}, A_{2} \in \mathbb{R}^{n \times n}$ respectively, the supra-adjacency matrix associated to $\mathcal{M}$ [1, 15] is the block matrix

$$
\mathbb{A}=\left(\begin{array}{cc}
A_{1} & I \\
I & A_{2}
\end{array}\right) \in \mathbb{R}^{2 n \times 2 n} .
$$

Once we have fixed some personalized vectors $\mathbf{v}_{1}, \mathbf{v}_{2} \in$ $\mathbb{R}^{n}$, we can define a new block matrix by associating to each layer $\ell_{i}$ a two-layer multiplex as follows:

$$
\mathbb{M}_{\mathbb{A}}=\frac{1}{2}\left(\begin{array}{ll}
\mathbb{M}_{1,1} & \mathbb{M}_{1,2} \\
\mathbb{M}_{2,1} & \mathbb{M}_{2,2}
\end{array}\right) \in \mathbb{R}^{4 n \times 4 n}
$$

where if $i=1,2$

$$
\mathbb{M}_{i, i}=\left(\begin{array}{cc}
\alpha P_{A_{i}} & (1-\alpha) I \\
2 \alpha I & (1-\alpha) \mathbf{e v}_{i}^{T}
\end{array}\right) \in \mathbb{R}^{2 n \times 2 n}
$$

corresponds to the connections between the physical layer and the teleportation layer of each $\ell_{i}$, while if $1 \leq i \neq$ $j \leq 2$

$$
\mathbb{M}_{i, j}=\left(\begin{array}{cc}
I & 0 \\
0 & (1-\alpha) \mathbf{e v}_{j}^{T}
\end{array}\right) \in \mathbb{R}^{2 n \times 2 n}
$$


corresponds to the cross connections between the physical layer and the teleportation layer of $\ell_{i}$ and $\ell_{j}$, since the first diagonal block gives the links between physical nodes of type $x \otimes \ell_{i}$ and $x \otimes \ell_{j}$ (following the notation used in $[1,15])$ while the second block matrix gives the links between the teleportation nodes by using the personalized vector $\mathbf{v}_{2}$. Different extensions of the idea of two-layer PageRank could be done in the multiplex settings but we have chosen this one because, as we shall see later on, it is guaranteed that a unique solution exists and this choice also allows to make some natural interpretations.

From $\mathbb{M}_{\mathbb{A}}$ another block matrix can be defined by reordering the blocks in such a way that all the physical layers appear first and later all the teleportation layers comes together as follows:

$$
M_{2}=\frac{1}{2}\left(\begin{array}{cc|cc}
\alpha P_{A_{1}} & I & (1-\alpha) I & 0 \\
I & \alpha P_{A_{2}} & 0 & (1-\alpha) I \\
\hline 2 \alpha I & 0 & (1-\alpha) \mathbf{e v}_{1}^{T} & (1-\alpha) \mathbf{e v}_{2}^{T} \\
0 & 2 \alpha I & (1-\alpha) \mathbf{e v}_{1}^{T} & (1-\alpha) \mathbf{e v}_{2}^{T}
\end{array}\right) .
$$

It is straightforward to check that all the spectral properties of $M_{2}$ are essentially the same as the corresponding $\mathbb{M}_{\mathbb{A}}$ since they are the same matrices after some blockpermutation (i.e. they are similar by using a block permutation matrix).

Now, by using a similar reasoning as used in the case of the monoplex networks (see previous section), $M_{2}$ defines a Markov chain in a multiplex network with two layers and therefore there always exists a unique vector $\hat{\pi}_{M} \in$ $\mathbb{R}^{4 n}$ such that

(i) $\hat{\pi}_{M}^{T}=\hat{\pi}_{M}^{T} M_{2}$ with $\hat{\pi}_{M}^{T} \mathbf{e}=2$.

(ii) If $\hat{\pi}_{M}^{T}=\left[\begin{array}{llll}\pi_{u 1}^{T} & \pi_{u 2}^{T} & \pi_{d 1}^{T} & \pi_{d 2}^{T}\end{array}\right]$ with $\pi_{u i}, \pi_{d i} \in \mathbb{R}^{n}$ for every $i=1,2$ then

$$
\begin{aligned}
& \pi_{u 1}^{T} \mathbf{e}=\pi_{u 2}^{T} \mathbf{e}=\beta, \\
& \pi_{d 1}^{T} \mathbf{e}=\pi_{d 2}^{T} \mathbf{e}=1-\beta,
\end{aligned}
$$

with $\beta=2 \alpha /(1+\alpha)$.

Note that $(i)$ holds since $M_{2}$ is row stochastic and primitive, while (ii) can be proved in a similar fashion as in the monoplex case shown in the previous section. In fact, by plugging $\hat{\pi}_{M}^{T}=\left[\begin{array}{llll}\pi_{u 1}^{T} & \pi_{u 2}^{T} & \pi_{d 1}^{T} & \pi_{d 2}^{T}\end{array}\right]$ into $\hat{\pi}_{M}^{T}=\hat{\pi}_{M}^{T} M_{2}$ we get the system of equalities

$$
\left.\begin{array}{l}
2 \pi_{u 1}^{T}=\pi_{u 1}^{T} \alpha P_{A_{1}}+\pi_{u 2}^{T}+2 \alpha \pi_{d 1}^{T} \\
2 \pi_{u 2}^{T}=\pi_{u 1}^{T}+\pi_{u 2}^{T} \alpha P_{A_{2}}+2 \alpha \pi_{d 2}^{T} \\
2 \pi_{d 1}^{T}=(1-\alpha)\left(\pi_{u 1}^{T}+\pi_{d 1}^{T} \mathbf{e v}_{\mathbf{1}}^{T}+\pi_{d 2}^{T} \mathbf{e v}_{\mathbf{1}}^{T}\right) \\
2 \pi_{d 2}^{T}=(1-\alpha)\left(\pi_{u 2}^{T}+\pi_{d 1}^{T} \mathbf{e v}_{\mathbf{2}}^{T}+\pi_{d 2}^{T} \mathbf{e v}_{\mathbf{2}}^{T}\right)
\end{array}\right\}
$$

Now, by multiplying the first equation by e we get

$$
2 \pi_{u 1}^{T} \mathbf{e}=\pi_{u 1}^{T} \alpha P_{A_{1}} \mathbf{e}+\pi_{u 2}^{T} \mathbf{e}+2 \alpha \pi_{d 1}^{T} \mathbf{e}
$$

that is

$$
2 \beta=\alpha \beta+\beta+2 \alpha(1-\beta)
$$

and this holds for

$$
\beta=\frac{2 \alpha}{1+\alpha}
$$

In a similar way it is straightforward to check that the rest of equations of (13) are satisfied for $\beta=2 \alpha /(1+\alpha)$.

The above properties allow to define the PageRank of a biplex in the following way.

Definition 3.1 Given a biplex network $\mathcal{M}=(\mathcal{N}, \mathcal{E}, \mathcal{S})$ of $n \in \mathbb{N}$, with layers $\mathcal{S}=\left\{\ell_{1}, \ell_{2}\right\}$ whose adjacency matrices are $A_{1}, A_{2} \in \mathbb{R}^{n \times n}$ respectively, two personalized vectors $\mathbf{v}_{1}, \mathbf{v}_{2} \in \mathbb{R}^{n}$ and damping factor $\alpha \in(0,1)$, the PageRank of the biplex $\mathcal{M}$ is the unique vector $\hat{\pi}_{2}$ given by

$$
\hat{\pi}_{2}=\frac{1}{2}\left(\pi_{u 1}+\pi_{u 2}+\pi_{d 1}+\pi_{d 2}\right) \in \mathbb{R}^{n} .
$$

Note that $\hat{\pi}_{2}^{T} \mathbf{e}=1$.

It is remarkable the fact that when the biplex network is defined by two equal layers $\ell_{1}=\ell_{2}=\ell$ and the conditions of Theorem 2.2 are satisfied, then the PageRank of the biplex network, given by Definition 3.1 coincides with the two-layer approach PageRank of a network given by $\ell$ obtained from Definition 2.1 as the following result holds:

Theorem 3.2 Given a biplex network $\mathcal{M}=(\mathcal{N}, \mathcal{E}, \mathcal{S})$ of $n \in \mathbb{N}$, with undirected layers $\mathcal{S}=\left\{\ell_{1}, \ell_{2}\right\}$ whose adjacency matrices are $A_{1}=A_{2}=A, \mathbf{d}=\mathbf{0}, \mathbf{v}_{\mathbf{1}}=\mathbf{v}_{\mathbf{2}}=$ $\mathbf{v}=\mathbf{e} / n$, and $k_{\text {out }}(i)=k_{\text {out }}(j)$ for all $i, j \in \mathcal{N}$ (i.e. the layers are regular graphs), then $\hat{\pi}_{2}=\hat{\pi}_{A}$.

Proof:

Note that the system of equalities (13) becomes

$$
\left.\begin{array}{l}
2 \pi_{u 1}^{T}=\pi_{u 1}^{T} \alpha P_{A}+\pi_{u 2}^{T}+2 \alpha \pi_{d 1}^{T} \\
2 \pi_{u 2}^{T}=\pi_{u 1}^{T}+\pi_{u 2}^{T} \alpha P_{A}+2 \alpha \pi_{d 2}^{T} \\
2 \pi_{d 1}^{T}=(1-\alpha)\left(\pi_{u 1}^{T}+\pi_{d 1}^{T} \mathbf{e v}^{T}+\pi_{d 2}^{T} \mathbf{e v}^{T}\right) \\
2 \pi_{d 2}^{T}=(1-\alpha)\left(\pi_{u 2}^{T}+\pi_{d 1}^{T} \mathbf{e v}^{T}+\pi_{d 2}^{T} \mathbf{e v}^{T}\right)
\end{array}\right\} .
$$

The proof consists in showing that the solution to this system is given by

$$
\begin{aligned}
& \pi_{u 1}=\pi_{u 2}=\pi_{u}=\frac{1}{n} \beta \mathbf{e} \\
& \pi_{d 1}=\pi_{d 2}=\pi_{d}=\frac{1}{n}(1-\beta) \mathbf{e}
\end{aligned}
$$

with $\beta=2 \alpha /(1+\alpha)$.

By plugging $\pi_{u 1}=\pi_{u 2}=\pi_{u}$ and $\pi_{d 1}=\pi_{d 2}=\pi_{d}$ into (15) we get the equivalent system

$$
\left.\begin{array}{l}
2 \pi_{u}^{T}=\pi_{u}^{T} \alpha P_{A}+\pi_{u}^{T}+2 \alpha \pi_{d}^{T} \\
2 \pi_{d}^{T}=(1-\alpha) \pi_{u}^{T}+2(1-\alpha) \pi_{d}^{T} \mathbf{e v}^{T}
\end{array}\right\},
$$

that simplifies to

$$
\left.\begin{array}{rl}
\pi_{u}^{T} & =\pi_{u}^{T} \alpha P_{A}+2 \alpha \pi_{d}^{T} \\
2 \pi_{d}^{T} & =(1-\alpha) \pi_{u}^{T}+2(1-\alpha) \pi_{d}^{T} \mathbf{e v}^{T}
\end{array}\right\} .
$$


It is a routine to show that $\pi_{u}=\beta \mathbf{e} / n$, and $\pi_{d}=$ $(1-\beta) \mathbf{e} / n$ is a solution to this system. Therefore, the PageRank of the biplex network is given by

$$
\begin{aligned}
\hat{\pi}_{2} & =\frac{1}{2}\left(\pi_{u 1}+\pi_{u 2}+\pi_{d 1}+\pi_{d 2}\right) \\
& =\frac{1}{2 n}(2 \beta \mathbf{e}+2(1-\beta) \mathbf{e}) \\
& =\frac{1}{n} \mathbf{e}=\hat{\pi}_{A} .
\end{aligned}
$$

Now if we take the general case of a multiplex network $\mathcal{M}=(\mathcal{N}, \mathcal{E}, \mathcal{S})$, with layers $\mathcal{S}=\left\{\ell_{1}, \cdots, \ell_{k}\right\}$ whose adjacency matrices are $A_{1}, \cdots, A_{k} \in \mathbb{R}^{n \times n}$ respectively and we fix some personalized vectors $\mathbf{v}_{1}, \cdots, \mathbf{v}_{k} \in \mathbb{R}^{n}$, we can define a new block matrix by associating to each layer $\ell_{i}$ a two-layer multiplex as follows:

$$
\mathbb{M}_{\mathbb{A}}=\frac{1}{2}\left(\begin{array}{cccc}
\mathbb{M}_{1,1} & \mathbb{M}_{1,2} & \cdots & \mathbb{M}_{1, k} \\
\mathbb{M}_{2,1} & \mathbb{M}_{2,2} & \cdots & \mathbb{M}_{2, k} \\
\cdots & \cdots & \cdots & \cdots \\
\mathbb{M}_{k, 1} & \mathbb{M}_{k, 2} & \cdots & \mathbb{M}_{k, k}
\end{array}\right) \in \mathbb{R}^{2 k n \times 2 k n}
$$

where if $1 \leq i \leq k$

$$
\mathbb{M}_{i, i}=\left(\begin{array}{cc}
\alpha P_{A_{i}} & (1-\alpha) I \\
2 \alpha I & (1-\alpha) \mathbf{e v}_{i}^{T}
\end{array}\right) \in \mathbb{R}^{2 n \times 2 n}
$$

corresponds to the connections between the physical layer and the teleportation layer of each $\ell_{i}$, while if $1 \leq i \neq$ $j \leq k$

$$
\mathbb{M}_{i, j}=\left(\begin{array}{cc}
I & 0 \\
0 & (1-\alpha) \mathbf{e v}_{j}^{T}
\end{array}\right) \in \mathbb{R}^{2 n \times 2 n}
$$

corresponds to the cross connections between the physical layer and the teleportation layer of $\ell_{i}$ and $\ell_{j}$.From $\mathbb{M}_{\mathbb{A}}$ another block matrix can be defined by reordering the blocks in such a way that all the physical layers appear first and later all the teleportation layers comes together as follows:

$$
M_{k}=\frac{1}{k}\left(\begin{array}{ll}
\mathbb{B}_{1,1} & \mathbb{B}_{1,2} \\
\mathbb{B}_{2,1} & \mathbb{B}_{2,2}
\end{array}\right) \in \mathbb{R}^{2 k n \times 2 k n},
$$

where

$$
\begin{gathered}
\mathbb{B}_{1,1}=\left(\begin{array}{cccc}
\alpha P_{A_{1}} & I & \cdots & I \\
I & \alpha P_{A_{2}} & \cdots & I \\
\cdots & \cdots & \cdots & \cdots \\
I & I & \cdots & \alpha P_{A_{k}}
\end{array}\right), \\
\mathbb{B}_{2,2}=(1-\alpha)\left(\begin{array}{ccc}
\mathbf{e v}_{1}^{T} & \cdots & \mathbf{e v}_{k}^{T} \\
\cdots & \cdots & \cdots \\
\mathbf{e v}_{1}^{T} & \cdots & \mathbf{e v}_{k}^{T}
\end{array}\right),
\end{gathered}
$$

$\mathbb{B}_{1,2}=(1-\alpha) I \in \mathbb{R}^{k n \times k n}$ and $\mathbb{B}_{2,1}=k \alpha I \in \mathbb{R}^{k n \times k n}$. Note that all the spectral properties of $M_{k}$ are essentially the same as the corresponding $\mathbb{M}_{\mathbb{A}}$ since they are the same matrices after some block-permutation, similarly to the biplex case.

The same reasoning used before proves that $M_{k}$ defines a Markov chain in a multiplex network with two layers and therefore there always exists a unique vector $\hat{\pi}_{M} \in$ $\mathbb{R}^{2 k n}$ such that

(i) $\hat{\pi}_{M}^{T}=\hat{\pi}_{M}^{T} M_{k}$ with $\hat{\pi}_{M}^{T} \mathbf{e}=k$.

(ii) If $\hat{\pi}_{M}^{T}=\left[\begin{array}{llllllll}\pi_{u 1}^{T} & \pi_{u 2}^{T} & \cdots & \pi_{u k}^{T} & \pi_{d 1}^{T} & \pi_{d 2}^{T} & \cdots & \pi_{d k}^{T}\end{array}\right]$ with $\pi_{u i}, \pi_{d i} \in \mathbb{R}^{n}$ for all $1 \leq i \leq k$, then

$$
\pi_{u i}^{T} \mathbf{e}=\gamma, \quad \pi_{d i}^{T} \mathbf{e}=1-\gamma,
$$

for all $i=1,2, \ldots, k$, with

$$
\gamma=\frac{k \alpha}{1+\alpha(k-1)}
$$

Once again, previous properties allow us to define the PageRank associated to a multiplex as follows:

Definition 3.3 Given a multiplex network $\mathcal{M}=$ $(\mathcal{N}, \mathcal{E}, \mathcal{S})$, with layers $\mathcal{S}=\left\{\ell_{1}, \cdots, \ell_{k}\right\}$ whose adjacency matrices are $A_{1}, \cdots, A_{k} \in \mathbb{R}^{n \times n}$ respectively, personalized vectors $\mathbf{v}_{1}, \cdots, \mathbf{v}_{k} \in \mathbb{R}^{n}$ and damping factor $\alpha \in(0,1)$, the PageRank of the multiplex network $\mathcal{M}$ is the unique vector $\hat{\pi}_{k}$ given by

$$
\hat{\pi}_{k}=\frac{1}{k} \sum_{j=1}^{k}\left(\pi_{d j}+\pi_{u j}\right) .
$$

Note that $\hat{\pi}_{k}^{T} \mathbf{e}=1$.

It is remarkable to point out that Theorem 3.2 can be extended for general multiplex networks as follows:

Theorem 3.4 Given a multiplex network $\mathcal{M}=$ $(\mathcal{N}, \mathcal{E}, \mathcal{S})$ of $n \in \mathbb{N}$, with undirected layers $\mathcal{S}=$ $\left\{\ell_{1}, \cdots, \ell_{k}\right\}$ whose adjacency matrices are $A_{1}=A_{2}=$ $\ldots=A_{k}=A, \mathbf{d}=\mathbf{0}, \mathbf{v}_{i}=\mathbf{v}=\mathbf{e} / n$ for all $i \in \mathcal{N}$, and $k_{\text {out }}(i)=k_{\text {out }}(j)$ for every $i, j \in \mathcal{N}$, then $\hat{\pi}_{k}=\hat{\pi}_{A}$.

Proof:

The procedure is similar to that of Theorem 3.2 . In the present case, we denote

$$
\pi_{u i}=\pi_{u}=\gamma \mathbf{e} / n, \quad \pi_{d i}=\pi_{d}=(1-\gamma) \mathbf{e} / n,
$$

for all $i \in \mathcal{N}$, with $\gamma=k \alpha /(1+\alpha(k-1))$. Then, it is easy to see that property $(i)$ stated before is equivalent to

$$
\left.\begin{array}{l}
k \pi_{u}^{T}=\pi_{u}^{T} \alpha P_{A}+(k-1) \pi_{u}^{T}+k \alpha \pi_{d}^{T} \\
k \pi_{d}^{T}=(1-\alpha) \pi_{u}^{T}+k(1-\alpha) \pi_{d}^{T} \mathbf{e v}^{T}
\end{array}\right\}
$$

and it is a routine to check that a solution is given by

$$
\pi_{u}=\frac{\gamma}{n} \mathbf{e}, \quad \pi_{d}=\frac{1-\gamma}{n} \mathbf{e} .
$$


Therefore, the PageRank of the multiplex network is

$$
\begin{aligned}
\hat{\pi}_{k} & =\frac{1}{k}\left(\sum_{i=1}^{k} \pi_{u i}+\sum_{i=1}^{k} \pi_{d i}\right) \\
& =\frac{1}{k n}(k \gamma \mathbf{e}+k(1-\gamma) \mathbf{e})=\frac{1}{n} \mathbf{e}=\hat{\pi}_{A} .
\end{aligned}
$$

Note that last Theorem states that the given definition of PageRank for a multiplex network consisting of the same layer $A$ repeated $k$ times collapses to the two-layer approach PageRank of $A$ when certain conditions hold (the same conditions imposed in Theorem 2.2, i.e. it must be an undirected network and all the nodes must have the same number of neighbours). Thus, the PageRank of the multiplex collapses to the usual PageRank in those conditions.

\section{NUMERICAL COMPARISON OF CENTRALITIES FOR THE MADRID METRO SYSTEM}

In this section we illustrate an application of the proposed model by considering the multilayer network composed of the lines of the Madrid metro system [18]. This system is formed by 12 line stations each of which is composed of a number of network stations (metro stops) ranging from 7 to 33 . The number of stations in each line is shown in Table II Lines 12 and 6 are cycle graphs while the rest of the lines are linear graphs. The total number of stations is 234 . Note that some stations are shared by different lines and this fact allows us to model the whole system as a multiplex formed by 12 layers. We assume that each layer is composed of 234 nodes. In each layer we consider real nodes (corresponding to actual stations of the line) and virtual nodes, (corresponding to stations that do not belong to the line). These virtual nodes allow us to model the whole system as a multiplex.

\begin{tabular}{|c|c|c|c|c|c|c|c|c|c|c|c|c|}
\hline Line \# & 1 & 2 & 3 & 4 & 5 & 6 & 7 & 8 & 9 & 10 & 11 & 12 \\
\hline Number of stations & 33 & 20 & 18 & 23 & 32 & 28 & 23 & 8 & 29 & 31 & 7 & 28 \\
\hline
\end{tabular}

TABLE II. Number of stations in each line of the Madrid metro.

In order to characterize a real node and a virtual node we apply the two following conditions:

- A real node must have a greater value of its corresponding personalization vector than a virtual node, independently of the layer: e.g., node number 1 corresponds to "Abrantes" which is a real station in layer Line 11 . This node must have a corresponding component of the personalization vector that is greater than any component of the personalization vector of any virtual node in any layer.

\begin{tabular}{|c|c|l|l|}
\hline Ranking & degree & Name of the station & Lines \\
\hline 1 & 7 & Avenida de América & $4,6,7,9$ \\
2 & 6 & Alonso Martínez & $4,5,10$ \\
& 6 & Vodafone Sol & $1,2,3$ \\
4 & 5 & Cuatro Caminos & $1,2,6$ \\
& 5 & Diego de León & $4,5,6$ \\
& 5 & Nuevos Ministerios & $6,8,10$ \\
& 5 & Plaza de Castilla & $1,9,10$ \\
8 & 4 & Argüelles & $3,4,6$ \\
& 4 & Bilbao & 1,4 \\
& 4 & Callao & 3,5 \\
& 4 & Canal & 2,7 \\
& 4 & Colombia & 8,9 \\
& 4 & Goya & 2,4 \\
& 4 & Gran Vía & 1,5 \\
& 4 & Gregorio Marañón & 7,10 \\
\hline
\end{tabular}

TABLE III. Top 15 ranking by degree

- If we compute the usual PageRank in a layer by using the personalization vector of that layer, then a real node must have a greater value of the corresponding component of the usual PageRank than any virtual node in that layer. This is to assure that a real node is ranked above a virtual node in each layer.

It can be checked that the two above conditions are satisfied by taking the component of the personalization vector of any real node as the constant value

$$
v_{\text {real }}=\frac{\alpha}{\max _{r}}
$$

where $\alpha$ is the usual parameter of Google's PageRank, and $\max _{r}$ is the maximum number of real nodes over all the layers. In this example, $\max _{r}=33$ since the maximum is attained at line 1 , as shown in Table III. Therefore, $v_{\text {real }}=0.85 / 33 \approx 0.0258$ in the computations presented in this paper. The rest of the components of the personalization vector in each layer are scaled such as all the components of the personalization vector sum up to 1 .

In order to complete the analysis the graph formed by the union of the 12 lines should be considered. This graph is called the projected graph of the multiplex network 1] and, in this case, it has 234 nodes. In order to serve as a basis for further comparisons we show in Table III the top-15 ranking by using the degree of the nodes in the projected graph. Note that nodes with degree 4 are sorted alphabetically (there are 24 nodes with degree 4). Note also that node "Avenida América" has degree 7 (and not 8) since there is a link (Avenida de América,Diego de León) shared between two lines.

Once this multiplex network is set, several centrality measures could be considered: the (classic) PageRank of 


\begin{tabular}{|c|l|l|l|}
\hline Ranking & PageRank & Name of the station & Lines \\
\hline 1 & 0.00959250 & Avenida de América & $4,6,7,9$ \\
2 & 0.00849797 & Vodafone Sol & $1,2,3$ \\
3 & 0.00829025 & Plaza de Castilla & $1,9,10$ \\
4 & 0.00787603 & Alonso Martínez & $4,5,10$ \\
5 & 0.00724492 & Cuatro Caminos & $1,2,6$ \\
6 & 0.00712225 & Oporto & 5,6 \\
7 & 0.00707473 & Mar de Cristal & 4,8 \\
8 & 0.00705510 & Pueblo Nuevo & 5,7 \\
9 & 0.00701733 & Legazpi & 3,6 \\
10 & 0.00701035 & Nuevos Ministerios & $6,8,10$ \\
11 & 0.00690138 & Pacífico & 1,6 \\
12 & 0.00670675 & Diego de León & $4,5,6$ \\
13 & 0.00642212 & Sainz de Baranda & 6,9 \\
14 & 0.00628778 & Guzmán el Bueno & 6,7 \\
15 & 0.00617953 & Colombia & 8,9 \\
\hline
\end{tabular}

TABLE IV. Top 15 ranking by usual PageRank of the projected graph

the projected graph (the usual analysis of centrality of a complex monoplex network), the proposed PageRank of the multiplex network and the aggregation of the (classic) PageRank of each layer (as an independent network). Note that PageRank-like centralities are not usually considered for transportation networks. Traditionally, the existence of teleportations in the PageRank model (given by the damping factor that models the random and instantaneous jump to any other node) has been considered as a drawback of these measures when transportation (or geometric) networks are analysed. Nevertheless, PageRank-like centralities can give valuable structural information about transportation and geometric networks, since each teleportation could be understood as the end/beginning of a travel along the network: Instead considering a unique random walker in an infinite sequence of time, we could understand the Markon chain as a family of a infinite number of random walkers in a finite sequence such that when a teleportation occurs, the walker end its journey and another walker starts in the destination of the teleportation.

If we take the projected (monoplex) graph with $n=$ 234 nodes, we can compute the usual PageRank by considering an homogeneous distribution of the personalization vector, that is $\mathbf{v}=\mathbf{e} / n$. Note that all the nodes in the projected graph are real nodes and therefore we don't need to handle virtual nodes. In this first numerical computations there is no reason for biassing the personalization vector to any node, but this could be considered for a more detailed analysis. The resulting top-15 ranking is shown in Table IV] Only 103 iterations were needed to obtain convergence with a tolerance for the stopping criterium of $t o l=10^{-10}$ (that is, the iterative method stops when the norm of the difference of two consecutive iterated vectors is lower than $t o l)$.

\begin{tabular}{|c|c|l|l|}
\hline Ranking & Multiplex PageRank & Name of the station & Lines \\
\hline 1 & 0.00584409 & San Bernardo & 2,4 \\
2 & 0.00570284 & Diego de León & $4,5,6$ \\
3 & 0.00565358 & Avenida de América & $4,6,7,9$ \\
4 & 0.00560449 & Canal & 2,7 \\
5 & 0.00557474 & Alonso Martínez & $4,5,10$ \\
6 & 0.00556924 & Tribunal & 1,10 \\
7 & 0.00551579 & Bilbao & 1,4 \\
8 & 0.00546684 & Núñez de Balboa & 5,9 \\
9 & 0.00544471 & Colombia & 8,9 \\
10 & 0.00544306 & Gran Vía & 1,5 \\
11 & 0.00542409 & Gregorio Marañón & 7,10 \\
12 & 0.00541039 & Argüelles & $3,4,6$ \\
13 & 0.00539591 & Callao & 3,5 \\
14 & 0.00535047 & Plaza de España & 3,10 \\
15 & 0.00528886 & Vodafone Sol & $1,2,3$ \\
\hline
\end{tabular}

TABLE V. Top 15 ranking by Multiplex PageRank

By using a linear regression we obtain that the values of the degree of each node correlate with the usual PageRank with a value of the squared coefficient of determination $r^{2}=0.808$. Furthermore, taking into account that both rankings have ties we also employ the Kendall coefficient $\tau$ with penalty parameter (see [5, 11, 20]) to perform this comparison. This value results to be $\tau=0.669$ and hence there is a soft correlation between degree and PageRank, as it was expected. The left panel of Figure2 shows the PageRank of the projected graph vs the degree of each node and it illustrates the correlation between these two centrality measures.

By using the multilayer model proposed in this paper and the personalization vectors explained at the beginning of this section we obtain the top-15 ranking shown in Table V 5988 iterations are needed in order to obtain convergence with the same stopping tolerance as before, tol $=10^{-10}$. In this example, the test spent about 30 seconds of real clock time in a standard computer.

By using linear regression we get that the values of the PageRank of the projected graph correlate with the multiplex PageRank with $r^{2}=0.278$. The value of the Kendall coefficient results to be $\tau=-0.0393$. The central panel of Figure 2 shows the PageRank of the projected graph vs multiplex PageRank of each node and it illustrates the correlation between these two centrality measures. Therefore, there is not a remarkable correlation between PageRank of the projected graph and the new Multiplex PageRank in this underground network.

In addition to this, the usual PageRank of each layer can be also computed. In each layer we consider the coexistence of real and virtual nodes and the personalization vector explained at the beginning of the section is used. Once the PageRank of each layer is computed, the mean value of the 12 corresponding values of the PageRank in each layer for each node is computed. The top-15 rank- 

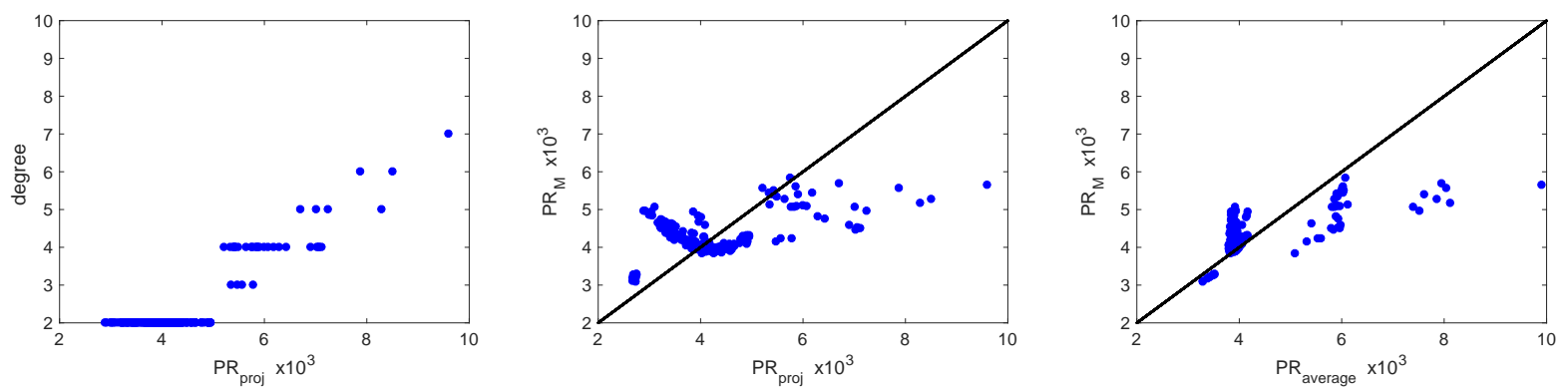

FIG. 2. PageRank of the projected graph vs the degree of each node (left panel), PageRank of the projected graph vs multiplex PageRank (central panel) and average PageRank vs multiplex PageRank(Right panel)

\begin{tabular}{|c|c|l|l|}
\hline Ranking & Average PageRank & Name of the station & Lines \\
\hline 1 & 0.00990651 & Avenida de América & $4,6,7,9$ \\
2 & 0.00811310 & Plaza de Castilla & $1,9,10$ \\
3 & 0.00803240 & Alonso Martínez & $4,5,10$ \\
4 & 0.00795143 & Diego de León & $4,5,6$ \\
5 & 0.00785430 & Vodafone Sol & $1,2,3$ \\
6 & 0.00760858 & Argüelles & $3,4,6$ \\
7 & 0.00751157 & Cuatro Caminos & $1,2,6$ \\
8 & 0.00738926 & Nuevos Ministerios & $6,8,10$ \\
9 & 0.00611087 & Chamartín & 1,10 \\
10 & 0.00606754 & San Bernardo & 2,4 \\
11 & 0.00603276 & Bilbao & 1,4 \\
12 & 0.00601649 & Canal & 2,7 \\
13 & 0.00601547 & Gran Vía & 1,5 \\
14 & 0.00600563 & Tribunal & 1,10 \\
15 & 0.00597868 & Colombia & 8,9 \\
\hline
\end{tabular}

TABLE VI. Top 15 ranking by average PageRank

ing of this average PageRank for each node is shown in Table VI.

By using a linear regression we obtain that the values of the average PageRank correlate with the multiplex PageRank with a value of $r^{2}=0.540$ and the value of the Kendall coefficient results to be $\tau=0.384$. The right panel of Figure[2]shows the average PageRank along each layer vs multiplex PageRank of each node and it illustrates the correlation between these two centrality measures. The results obtained ensure that there is a very low correlation between average (classic) PageRank along each layer and the new Multiplex PageRank proposed in this paper.

Previous numerical comparisons shows that the proposed multiplex PageRank constitutes a new centrality measure not strongly correlated with the classic PageRank or the averaged PageRank, but some correlations with other centrality measures could be expected. Let us finally consider the comparison with the betweenness centrality, since this is a usual parameter in transportation networks analysis. In [10] the author carried out a computation of the betweenness centrality for some Metro systems. In particular, the top-5 ranking obtained for Madrid metro is the following:

1. Avenida de América,

2. Vodafone-Sol,

3. Plaza de España,

4. Pacífico,

\section{Alonso Martínez.}

Notice that two of these stations are among the top- 5 ranking given by the multiplex PageRank and 4 of these stations are among the the top-15 ranking given by the multiplex PageRank (see Table V). The Pacífico station (with stops at lines 1 and 6 ) is ranked with multiplex PageRank at position 47. Once again, multiplex PageRank offers a different ranking compared with rankings obtained with known centrality measures.

\section{CONCLUSIONS}

Understanding the usual PageRank of a complex (monoplex) network allows to introduce a new centrality measure $\hat{\pi}_{A}$ that has several remarkable properties, such as

- It preserves convergence properties of usual PageRank.

- $\hat{\pi}_{A}$ equals to usual PageRank for undirected regular graphs, with no dangling nodes.

- Numerical experiments show that $\hat{\pi}_{A}$ correlates quite well with usual PageRank.

- Despite that the iteration matrix $M_{A}$ has a dense block $\mathbf{e v}^{T}$, there's no need to compute it explicitly. That is, matrix $M_{A}$ is well suited for iterative computations. 
In addition to this, the biplex approach to the PageRank allows to present a proposal for calculating the PageRank of a multiplex that is based on an original interpretation of the usual PageRank. Hence, an ad-hoc PageRank adapted to the formulation of multiplex networks has been introduced. This definition is well suited for numerical computations and has an easy interpretation. If this new multiplex PageRank is denoted by $\hat{\pi}_{k}$, some analytical results have been proved and the most outstanding features of this new multiplex formulation are the following:

- Convergence is assured.

- Numerical computations can be carried out without the use of dense matrices.

- In some limit cases the PageRank of the multiplex coincides with the usual PageRank.

An illustration of this new model is presented by analysing the centrality of the Madrid underground public system that allowed to show that the multiplex PageRank is a new centrality measure not significantly correlated with other centrality measures of multiplex networks.

\section{ACKNOWLEDGEMENTS}

This work has been partially supported by the project MTM2014-59906 (Spanish Ministry) and the grant URJC-Grupo de Excelencia Investigadora GARECOM (2014-2016).
[1] S. Boccaletti, G. Bianconi, R. Criado, C.I. del Genio, J. Gómez-Gardeñes, M. Romance, I. Sendiña-Nadal, Z. Wang and M. Zanin, The structure and dynamics of multilayer networks Physics Reports 544(1) (2014), 1122.

[2] S. Boccaletti, V.Latora, Y. Moreno, M. Chavez and D.U.Hwang, Complex Networks: Structure and Dynamics, Phys. Rep, 424 (2006), 175.

[3] M. Boguñá, R. Pastor-Satorras, A. Díaz-Guilera and A. Arenas Physical Review E 70 (2004), 056122.

[4] R. Bru, F. Pedroche and D.B. Szyld, Cálculo del vector PageRank de Google mediante el método aditivo de Schwarz (In spanish). Proc. of. Congreso de Métodos Numéricos en Ingeniería 2005. J. L. Pérez Paracio et al, (eds). SEMNI.

[5] R. Criado, E. García, F. Pedroche and M. Romance, A new method for comparing rankings through complex networks: Model and analysis of competitiveness of major European soccer leagues, Chaos 23 (2013), 043114. doi:10.1063/1.4826446

[6] M. De Domenico, A. Solé-Ribalta, E. Cozzo, M. Kivela, Y.Moreno, M.A. Porter, S. Gómez and A. Arenas, Mathematical formulation of multi-layer networks, Phys. Rev. X 3 (2013), 041022.

[7] M. De Domenico, A. Solé-Ribalta, S. Gómez and A. Arenas, Navigability of interconnected networks under random failures, PNAS, 111 (23) (2013), 8351-8356. doi:10.1073/pnas.1318469111

[8] M. De Domenico, A. Solé-Ribalta, E. Omodei, S. Gómez and A. Arenas, Ranking in interconnected multilayer networks reveals versatile nodes, Nature Communications 6 (2015),6868. doi:10.1038/ncomms7868

[9] A.Solé-Ribalta, M. De Domenico, S. Gómez and A. Arenas Random walk centrality in interconnected multilayer networks, To appear in Physica D (2016).

[10] S.Derrible, Network Centrality of Metro Systems, PLoS ONE, 7(7) (2012), e40575. doi:10.1371/journal.pone.0040575

[11] R. Fagin, R. Kumar, M. Mahdian, D. Sivakumar and E.Vee, Comparing Partial Rankings, SIAM J. Discrete Math., 20(3) (2006), 628-648.
doi:10.1137/05063088X

[12] S. Fortunato, M. Boguñá, A. Flammini and F. Menczer, Approximating PageRank from In-Degree, Lecture Notes in Computer Science 4936 (2008), 59-71.

[13] E. García, F. Pedroche and M. Romance, On the localization of the Personalized PageRank of Complex Networks, Linear Algebra and its Applications 439 (2013), 640-652.

[14] A. Halu, R.J. Mondragón, P. Panzarasa and G. Bianconi, Multiplex PageRank, PLoS ONE 8 (10) (2013).

[15] M. Kivelä, A. Arenas, M Barthelemy, J.P. Gleeson, Y.Moreno and M.A.Porter, Multilayer networks, Journal of Complex Networks 2 (3) (2014), 203-271.

[16] S.D. Kamvar, T.H. Haveliwala and G.H. Golub, Adaptive methods for the computation of PageRank, Linear Algebra and its Applications 386 (2004), 51-65.

[17] M.G. Kendall, A New Measure of Rank Correlation, Biometrika $30 \quad(1-2) \quad$ (1938), 81-89. doi:10.1093/biomet/30.1-2.81

[18] http://www.metromadrid.es/

[19] L. Page, S. Brin, R. Motwani and T. Winograd The PageRank citation ranking: Bridging order to the Web Tech.Rep. 66, Stanford University. 1998.

[20] F. Pedroche, R.Criado, E. García, M. Romance and V.E. Sánchez, Comparing series of rankings with ties by using complex networks: An analysis of the Spanish stock market (IBEX-35 index), Networks Het.Media 10 (1) (2015), 101-125. doi:10.3934/nhm.2015.10.101

[21] M. Romance, L. Solá, J. Flores, E. García, A.J. García del Amo and R. Criado, A Perron-Frobenius theory for block matrices associated to a multiplex network, Chaos, Solitons and Fractals 72 (2015) 77-89.

[22] L. Solá, M. Romance, R. Criado, J. Flores, A. García del Amo and S. Boccaletti, Eigenvector centrality of nodes in multiplex networks, Chaos 23 (2013), 033131.

[23] A. Solé-Ribalta, M. De Domenico, S. Gómez and A. Arenas, Centrality rankings in multiplex networks, Proceedings of the 2014 ACM Web Science Conference (2014), 149-155.

[24] A.L. Traud, E.D. Kelsic, P.J. Mucha, and M.A.Porter, Comparing Community Structure to Characteristics in Online Collegiate Social Networks SIAM Rev.53 (3) 
(2011), 526-543. doi:10.1137/080734315

[25] R.S. Varga, Matrix Iterative Analysis (2nd. Ed.), Springer, Berlin (2000).
[26] S. Wasserman and K. Faust, Social Networks Analysis. Cambridge University Press, London (1994). 